\title{
Alojamiento de Bajo Impacto Ambiental en la Patagonia Argentina
}

\author{
Regina G. Schlüter
}

RESUMEN: Análisis de las diferentes formas de alojamiento de bajo impacto ambiental que han cobrado vigencia al popularizarse el ecoturismo. Se finaliza con una referencia al turismo en la Patagonia Argentina y con un análisis de algunos tipos de alojamiento que han surgido durante la última década con el objeto de satisfacer las necesidades de los turistas que buscan un contacto más estrecho con la naturaleza.

PALABRAS CLAVE: Turismo e ecologia; ecoturismo; alojamento; "ecolodges"; Patagonia; Argentina.

ABSTRACT: The article analyzes different environmental impact lodging emerged with the spread of ecolourism. Finally, refers to tourism in Argentinean Patagonia analyzing some kind of lodges appeared during last decade aiming to satisfy those lourists who search a closer contacl wilh nature.

KEY WORDS: Tourism and ecology; ecolourism; lodging; ecolodges; Palagonia; Argentina.

\section{Introduccion}

La preocupación por la conservación del medio ambiente no se reduce al lugarde residencia habitual de las personas, sino que también se ha extendido hacia los lugares receptores de turismo.

La industria turística en general, y la del alojamiento en particular, no ha permanecido ajena a la preferencia de susclientes de permanecer durante su período vacacional en un ambiente ordenado y poco contaminado.

El sector alojamiento ha tomado una serie de medidas para preservar el medio. Entre ellas se puede mencionar: evitar la contaminación en todas sus 
formas, ahorrar energía y consumo de agua, utilizar productos locales para la claboración de alimentos, adoptar un estilo arquitectónico en armonía con el paisaje, etc. (Boers \& Bosch, 1994).

Asimismo, paralclamente a la popularización del término ecoturismo para señalar una forma de turismo en armonía con el medio, se comenzó a llamar "ecolodges" a las diferentes formas de alojamiento cuya filosofia de diseño y operación se ajustaban a un turismo basado en la naturaleza y que producían un bajo impacto negativo en el medio fisico y humano en el que se localizaban.

\section{"Ecolodges" - Alojamiento de Bajo Impacto Ambiental}

El término "ecolodge" se comenzó a popularizar en los países o regiones que buscaron posiccionarse en el mercado como destino para el ecoturismo, como por cjemplo, Costa Rica, la Amazonía, Sudáfrica, y Africa oriental.

A partir de 1994, el tema del diseño de los "ecolodges" se convirtió en el favorito de congresos y publicaciones científicas relacionadas con el ecoturismo. Asimismo, con miras a la captación de un mercado específico, se han denominado "ecolodges" a diferentes formas de alojamiento que abarcan zonas climáticas tan opuestas como cl ártico y cl trópico.

Russell, Bottril \& Meredith (1995) señalan que hasta la fecha no existe un criterio estandarizado respecto a qué es un "ccolodge", pero identificaron doze características que a su juicio lo diferencian de un "lodge" tradicional (Cuadro l).

Sin embargo, Rushmann (1992:139) afirma que en el Amazonas Brasileño

los "lodges" - medios de hospedaje del turismo ambiental y ecológico - están bajo riguroso control de las autoridades turisticas y ambientalistas que, a través de una legislación especifica, procuran integrar los proyectos arquitectónicos al paisaje local valorizando su exotismo pero evitando la agresión a los ecosistemas.

Los lodges se construyen en áreas densamente arboladas -distantes algunas horas de navegación de las principales ciudades del Amazonas-y próximas a correntadas, lagos o ríos menores; están equipados con radio-teléfono para comunicación de emergencia con las ciudades más próximas. (...) La electricidad para el funcionamiento de ventiladores, refrigeradores, luces, etc. la proveen equipos generadores pero, debido a que el ruido de los motores se contrapone al silenco o a los sonidos tipicos del bosque, la mayoria de los lodges los utilizan lo menos posible.

Selengunt (1995), basándose en su cxperiencia en el diseño de un complejo turístico en Maho Bay (Islas Vírgenes de los EUA), pone énfasis en la mimetización de las construcciones con el paisaje; la utilización de energía renovable y en una serie de normas de preservación ambientales muy similares a los que Accor Management Limited utiliza para sus hoteles de la cadena Novotel (ButterviorthHeinemann, 1993), es decir, las "5 Rs" de Repensar, Re-utilizar, Reducir. Racionalizar, Reciclar, y Recuperar.

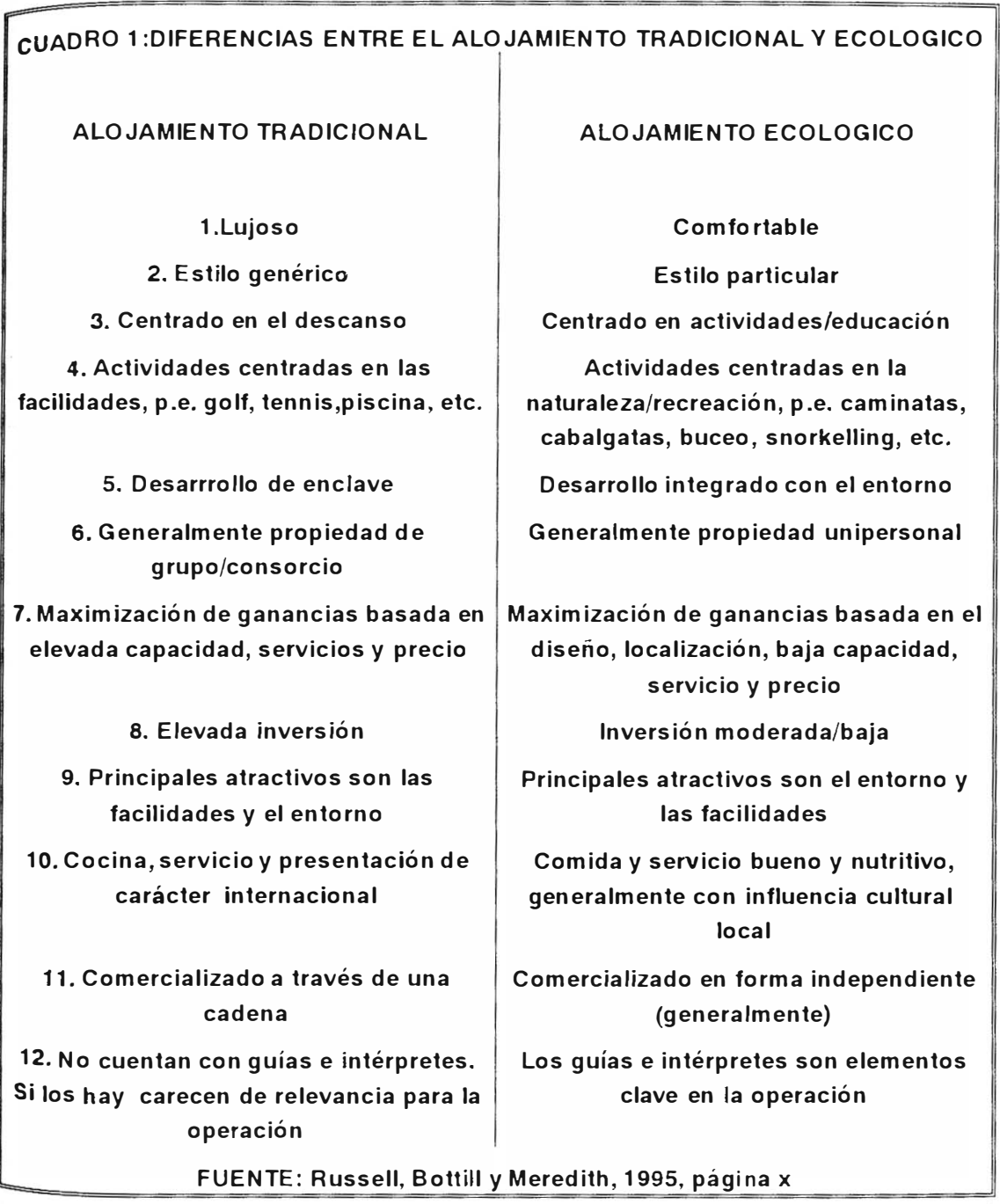


Costa Rica, uno de los principales destinos ecoturísticos del mundo, coloca el acento en los aspectos cdilicios del alojamicnto, cuya altura no deberá superar a la de una palmera para, de esta forma, mimetizarse con el paisaje (Schlüter, 1992)

En los principales destinos turísticos del Africa como, por cjemplo, Rep. de Sudáfrica, Kenia y Tanzania son populares los "tented camps", cuya traducción literal es "campos entoldados". Consisten, básicamente, en carpas/habitaciones semipermanentes ubicadas en lugares estratégicos para que no interfieran con la vista y cn un centro de atención al visitante, integrado generalmente, por una oficina administrativa, sanitarios, tienda, bar, comedor y espacio para fogatas nocturnas. Las instalaciones del centro de atención al visitante están hechas con el mismo material que las carpas o con clementos disponibles en cl área.

Los complejos se encuentran cercados para evitar que se aproximen animalcs salvajes. Asimismo, por razones de scguridad, no se permite dejar elementos fucra de los espacios cerrados. De esta manera se evita tambićn la tentación de poner a secar prendas de vestir recićn lavadas o tirar desperdicios en lugares de uso común. Si la afluencia turística disminuye a cifras tales que no se considera rentable su cxplotación los complejos son desmantelados - el proceso, al igual que el de instalación es de aproximadamente tres semanas - y sólo quedarán delgadas capas de cemento en los lugares que alguna vez estuvieron cubicrtos, cemento que las inclemencias del ticmpo - cn especial durante la temporada de lluvias - prontamente destruirán (Schlüter, 1993)

Galbreath (1995) señala que en los EUA se fabrican "kits" de "ccolodges" que se cxportan a cualquicr parte del mundo. Para adaptarse a los diferentes ambientes culturales se presentan en tres diferentes diseños. Consisten en sistemas modulares autosuficentes que generan su propia electricidad, regulan la temperatura de los ambientes internos y purifican las aguas servidas. Solamente precisan de una conexión cxtcrna para contar con agua corriente.

\section{Turismo y Ecologia en la Patagonia Argentina}

El turismo en la Patagonia Argentina siempre cstuvo cstrechamente ligado a su patrimonio natural. Bariloche, el principal y a la vez más antiguo centro turístico de la región formó parte del Parque Nacional Nahuel Huapi hasta su desafectación en 1958. Las otras tres más importantes localidades turísticas del área cordillerana: San Martín de los Andes (Ncuquén), Esquel (Chubut) y El Calafate (Santa Cruz) funcionan como centro de servicios para los parques nacionales Lanin, Los Alerces y Los Glaciares respectivamente.

El turismo en la costa patagónica tomó gran impulso a partir de la creación del Sistema de Reservas Provinciales de la Provincia de Chubut. La variedad de fauna marina que se encuentra en la península Valdés y la pingüinera de Punta Tombo hicieron de Puerto Madryn, ubicada sobre el Golfo Nuevo, uno de los más importantes destinos para el turismo internacional.

El alojamiento que se construía con la intención de satisfacer las necesidades de los turistas tuvo en cuenta el perfil cconómico de los mismos y el estilo arquitectónico imperante en la época. En el área de los dos parques nacionales próximos, Nahuel Huapi y Lanín, se prefirió un estilo tipo alpino dado que se percibía el espacio como muy similar al de los Alpes europeos. En los restantes centros se privilegió un estilo de planta rectangular.

Sin embargo, en la última década tomaron gran auge las cabañas de madera con techo a dos aguas - cspecialmente apto para zonas de nevadas intensas. Estas cabañas, con servicios independientes (cocina, baño, estar y dormitorios) tienen una serie de ventajas: son cconómicas, son prefabricadas - por lo cual su instalación es rápida y sencilla -, su capacidad de aloj

personas - númcro promedio del grupo que viaja - y permiten aumentar la capacidad de alojamiento según la demanda y la disponibilidad de recursos del empresario (si el negocio funciona se van adquiriendo nuevas y al mismo tiempo continua en operación cl resto). Estas ventajas, aunado a queel estilo arquitectónico es percibido por la población local como atractivo para los turistas, ha determinado que se construyan cabañas, incluso en sitios desérticos en los que tanto los árboles como la nieve son prácticamente nulos.

El crecimiento del turismo en la Patagonia sufrío un largo retraso debido a la distancia que scpara a la región de los principales centros emisores, lo cual encarecia el desplazamiento y, a su vez, incidia negativamente en la construcción de facilidades para atender a los turistas.

El mejoramiento de la red caminera y la popularización del transporte aćreo, aunado al interés tanto del mercado interno como internacional por visitar lugares alejados, influyó positivamente en el turismo hacia la Patagonia. La construcción y el mejoramiento de la infraestructura vial entre Argentina y Chile, la facilitación delas formalidades fronterizas entreambospaises, y la finalización dela construcción de la Carretera Austral en Chile - que permite apreciar paisajes de gran belleza son factores que se suman para atraer turistas a la región.

Si bien la Patagonia aparenta ser culturalmente uniforme, la realidad muestra tres zonas claramente diferenciadas: nortc, centro y sur. Esta diferencia cultural se refleja en la respuesta dada en el momento de crear las facilidades para atender a los turistas, particularmente en lo que respecta al alojamiento localizado fuera de las grandes concentracciones urbanas.

En la Patagonia Norte, tambićn conocida como la Región de Los Lagos, predominaron las hosterias. A grandes rasgos se ajustan a los "lodges" del Cuadro 1 y se categorizan por estrellas (de 1 a 3 estrellas). En su mayoría se encuentran próximas a los cascos urbanos por lo cual se conectan a la red de luz, agua potable cloacas y recolección de basura. Cuando no cuentan con alguno de estos servicios utilizan grupos electrógenos y los medios tradicionales para la obtención de agua y desecho de residuos sólidos y líquidos (pozos negros, separación de basura biodegradable de aquella no degradable, colocando a la primera en lugares profuntos y reutilizando a la segunda. El agua se obtiene por bombco y se extrae de lagos o de napas subterráneas que se encuentran a mayor profundidad que los pozos negros. 
Fotografía 1: Cabaña y lago Vintter
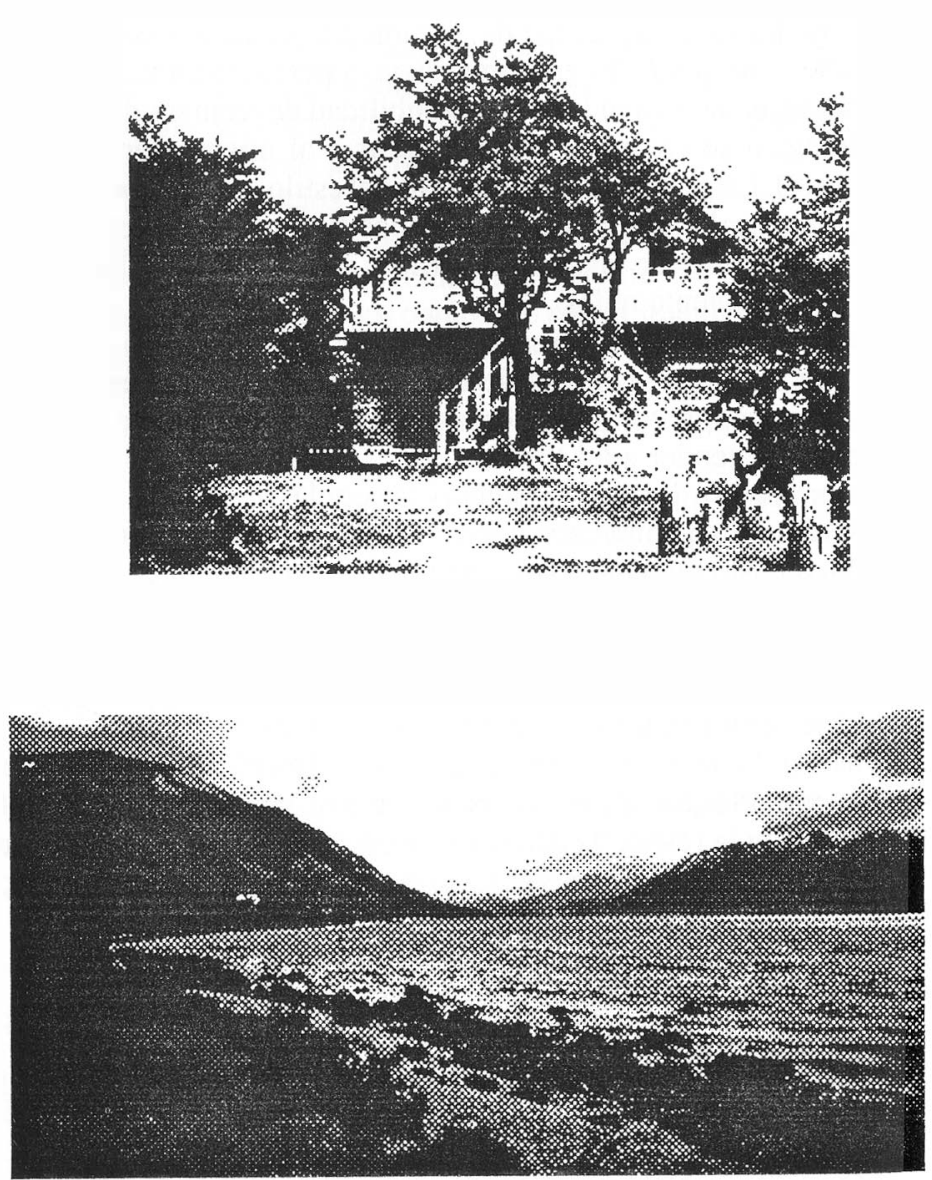

En la Patagonia Austral se desarrolló un producto denominado estancias turisticas. Consiste básicamente en el reacondicionamiento de las casonas donde residen los propietarios de grandes cxtensiones de campo dedicadas a la cría del ganado ovino. En algunos casos se privilegia el alojamiento. En otros una serie de actividades relacionadas con las tareas rurales tradicionales y con el avistaje de fauna de la zona.

En el Centro de la Patagonia Argentina sc encuentra cnclavada en la cordillera de los Andes una serie de lagos de gran belleza y alejados de los principales centros urbanos de la zona. Debido a que las fuertes nevadas aislan a los lagos durante los largos inviernos cuentan con una infraestructura que se limita aalgún puesto de Gendarmería Nacional o a la vivienda de un "puestero" (cuidador de ovejas). El auge del ecoturismo ha determinado que en los últimos años se iniciara la construcción de "ecolodges".

A este tipo de alojamiento sc lo denomina comúnmente cabañas y es de reciente data. Dado que se localiza en áreas donde la naturaleza constituye el atractivo principal, se toman diferentes recaudos para interferir lo menos posible con ella. A continuación se presentarán tres ejemplos que constituyen los tipos básicos que se encuentran en la región.

\section{Cabañas Lago Vintter}

Se cncuentran a orillas del lago del mismo nombrc. Se concecta con el lago Palena en Chilc y su principal caracteristica es su gran aleaje debido a los fuertes vientos de la región. Durante la temporada que se extiende entre noviembre y abril atrae a gran cantidad de pescadores, tanto del pais como del extranjero. Muy próximo al lago Vintter se encuentran otros dez lagos con buena pesca. Durante la temporada 1995/94 funcionó una sóla cabaña. Su número se amplió a dos en la temporada 1995/96. Cada cabaña está diseñada para albergar confortablemente a seis personas. La principal característica de las cabañas es su tipo constructivo, ya que se mimetiza totalmente con el bosque que la rodea (Fotografia 1). No cuenta con luz artificial y el combustible que se utiliza para cocinar y calefaccionar los ambientes proviene de los alrededores. Dado que la clientela se dedica a la pesca ecológica (con devolución de las piezas excepto las que puede consumir en el día), toda su operación está orientada a csa actividad.

\section{Cabaña Huente-Có}

Este pequeño complejo se encuentra a orillas del lago La Plata, en el sudoeste de la provincia de Chubut. Se encuentra en operación desde unos diez años aproximadamente y cs una de las más antiguas de la región. En sus proximidades se encuentran cotos de cazade ciervo colorado y cl lago es bueno para la pesca. En un principio la clientela se dedicaba a cazar y pescar pero, con el fortalecimiento de la conciencia ecológica y cl interés por conocer mejor el área, sc fue modificando 
Fotografía 2: Exterior e interior de Cabaña Huente-Có
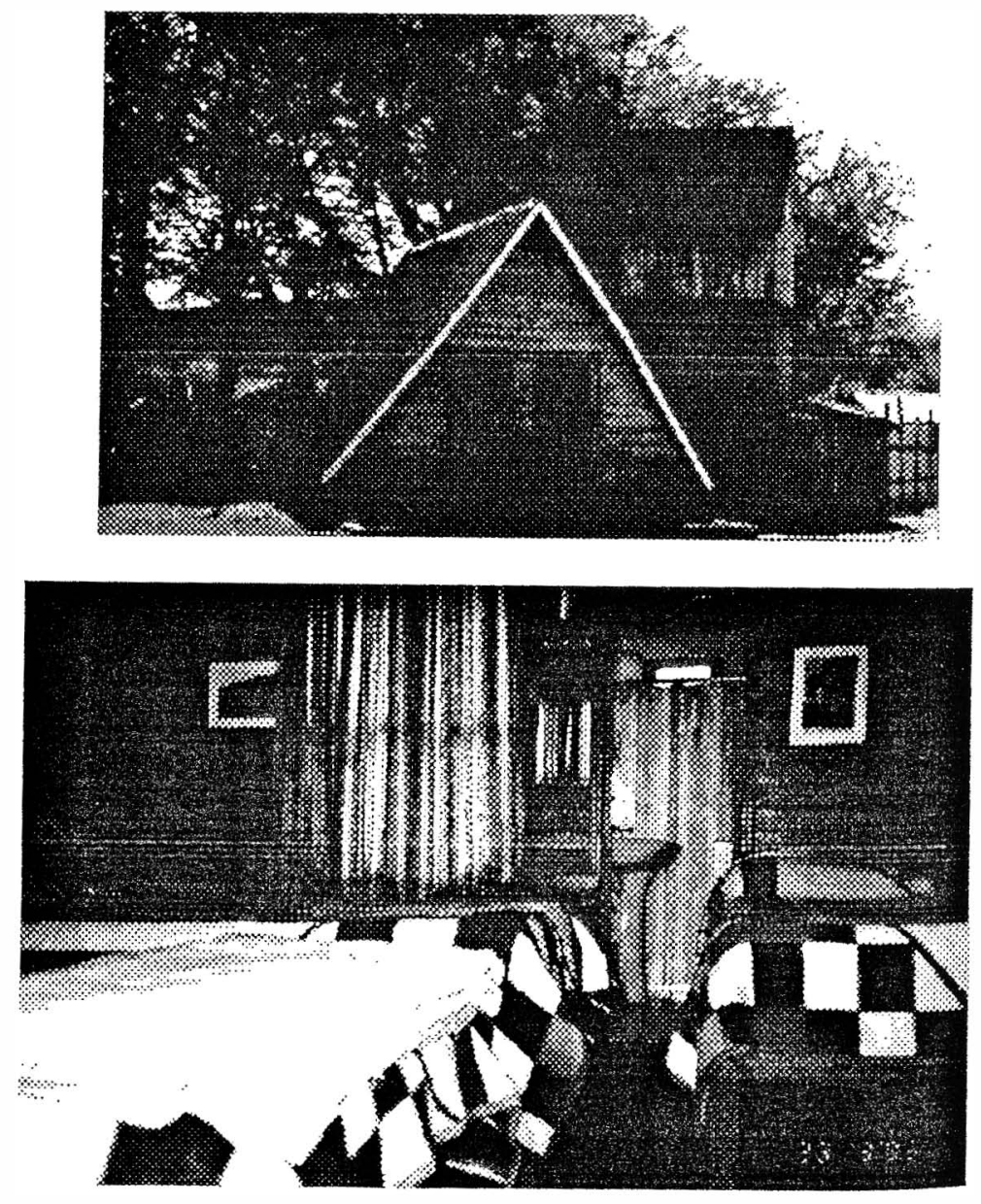

lentamente su perfil. Actualmente se privilegian los paseos en bote, largas caminatas y cscalar las cerros que rodean al lago. La capacidad de alojamiento sc reduce a un par de cabañas con $4-6$ camas y baño (Fotografia 2). La casa principal que hace a la vez de comedor, cocina y salón social, se transforma en un amplio dormitorio cuando llegan estudiantes y cstudiosos universitarios de diversas disciplinas para realizar trabajos de campo. Cuenta con gencrador electrógeno y el combustible para calefaccionar y cocinar es la leña proveniente de los bosques cercanos.

\section{Complejo Turístico Las Margaritas}

Se encuentra a orillas del lago del mismo nonbre muy próximo a la frontera con Chilc. Inició sus operaciones durante la temporada 1993/94 y está cn proceso de expansión. Actualmente consta de cuatro cabañas y un gran salón para realizar diversas actividades sociales. Las cabañas son de dos plantas y con una capacidad para seis personas. Además de los dormitorios y baño cuentan con cocina y cstar

Este complejo se diferencia de los restantes en la Patagonia porque fue construido considerando diversos elementos de preservación ambiental. Cuenta con un sistema de tratamiento de aguas servidas. El agua potabla proviene del lago y es analizada constantemente para constatar su purcza. Los alimentos son producidos en cl lugar. Debido a las inclemencias del tiempo se ha construído un invernáculo que provec de verduras y cspecias (Fotografia 3). La luz se genera con un grupo electrónego pero para mantener su consumo al mínimo sc ha construido una cámara bajo tierra donde las bebidas y alimentos perecederos se mantiene naturalmente refrigerados. La basura se separa y aquella que no es biodegradable se recicla o se transporta fuera. Como combustible para cocinar y calefaccionar se utiliza leña del bosque cercano. Las actividades cstán orientadas a un mejor conocimiento del patrimonio natural y cultural que rodea al complejo

\section{Conclusion}

La Patagonia Argentina cuenta con grandes atractivos naturales y paisajes culturales de gran belleza y fragilidad. Estos fueron los que posibilitaron el inicio del turismo hacia la región y en la actualidad atraen cada vez a un mayor número de visitantes provenientes tanto del país como del cxtranjero.

Si bien desde el principio se tuvo en claro que para que la región no perdiera su encanto habia que proteger la naturaleza, no se tomaron en cuenta determinados elementos para evitar la contaminación proveniente de las facilidades para satisfacer las necesidades de los turistas.

La idea gencralizada continúa siendo que manteniendo bajo cl número de comidades también se manticne bajo cl ricsgo de contaminación. Sin embargo, a pesar de que los esfuerzos promocionales no son intensos, continúa aumentando la afluencia espontánea de turistas que también comenzarán a demandar productos 
Fotografía 3: Cabaña e invernáculo del Complejo Turístico Las Margaritas
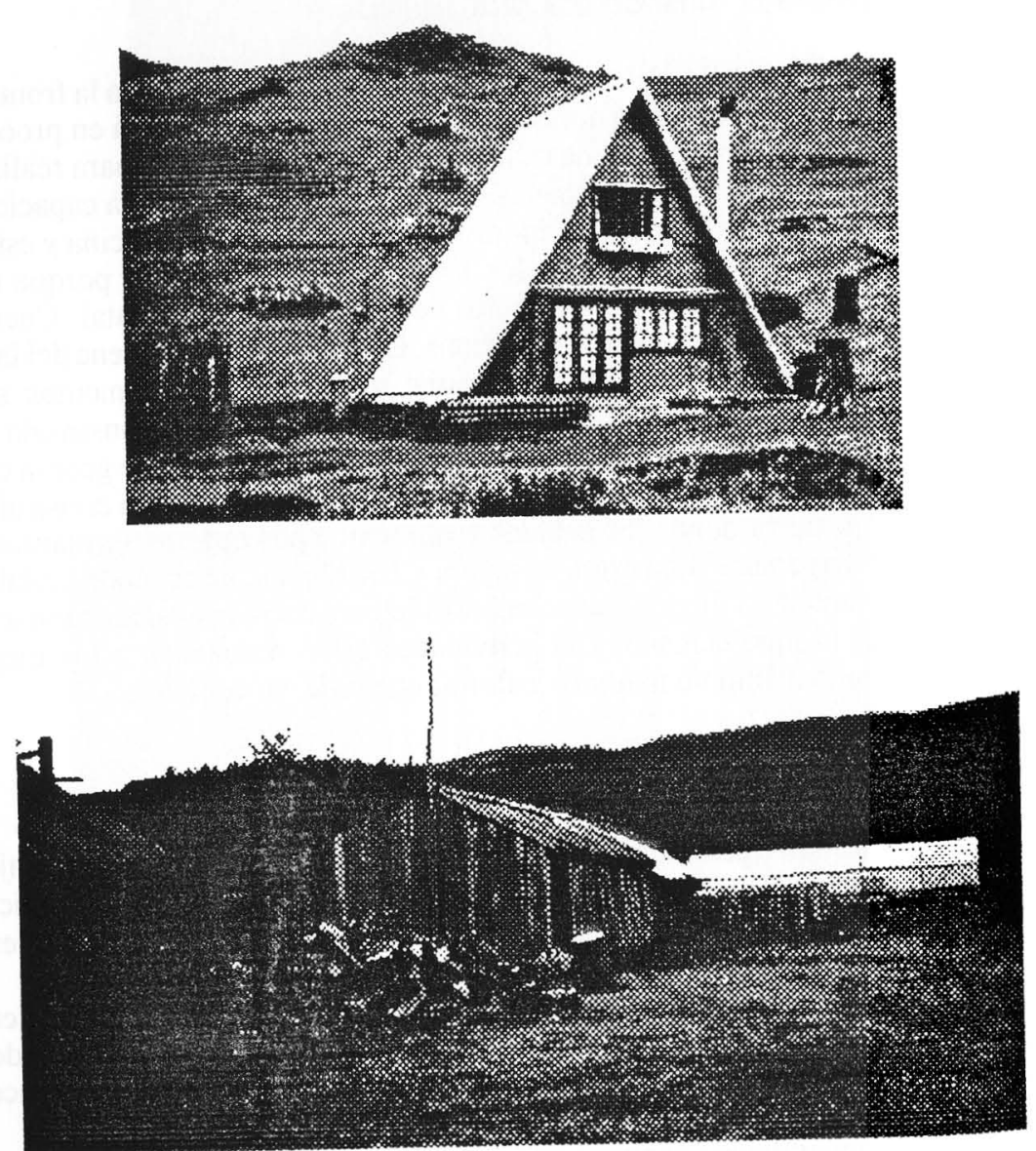

más sofisticados. Para satisfacer esas necesidades y no impactar en el ambiente es necesario que hoy se comiencen a considerar a las nuevas tecnologías que permiten ofrecer una clevada calidad en el servicio y a la vez preservar el entorno.

\section{BIBLIOGRAFIA}

BOERS, H.; BOSCH, M. 1994. The earth as a holiday resort. An introduction to tourism and the environment. Utrech SME/Institute for Environmental Communication and Netherlands Institute of Tourism and Transport Studies. BUTTERWORTII - HEINEMANN, 1993. Environmental management for hotels. The industry guide to best practice. Oxford: Butterworth Heineman

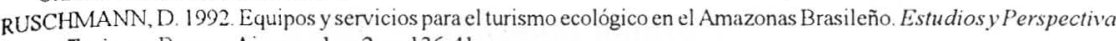

列 of for planners \& developers. North Bennington: The Ecotourisn

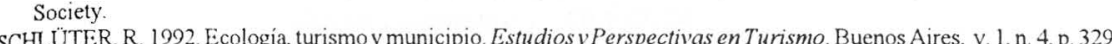

1993. Una forma de alojamiento de escaso impacto ambiental. Los "tented camps" de Kenia. Estudiosy Perspectiveas en Turismo. Buenos Aires, v. 2, n. 2, p. 172-9.

SELENGUT, S. 1995. Foreword. In: HAWKINS, D.E.; WOOD, M. E.; BITTMAN, S. (ed.). The ecolodge sourcebook for planners \& dev'elopers. Norh Bennington: The Ecotourism Sociely. 